\title{
Direct impact of cisplatin on mitochondria induces ROS production that dictates cell fate of ovarian cancer cells
}

\author{
Markus Kleih¹, Kathrin Böpple', Meng Dong ${ }^{1}$, Andrea Gaißler ${ }^{1}$, Simon Heine ${ }^{1}$, Monilola A. Olayioye², \\ Walter E. Aulitzky ${ }^{3}$ and Frank Essmann ${ }^{1}$
}

\begin{abstract}
Patients with high-grade serous ovarian cancer (HGSC) frequently receive platinum-based chemotherapeutics, such as cisplatin. Cisplatin binds to DNA and induces DNA-damage culminating in mitochondria-mediated apoptosis. Interestingly, mitochondrial DNA is critically affected by cisplatin but its relevance in cell death induction is scarcely investigated. We find that cisplatin sensitive HGSC cell lines contain higher mitochondrial content and higher levels of mitochondrial ROS ( $m$ tROS) than cells resistant to cisplatin induced cell death. In clonal sub-lines from OVCAR-3 mitochondrial content and basal oxygen consumption rate correlate with sensitivity to cisplatin induced apoptosis. Mitochondria are in two ways pivotal for cisplatin sensitivity because not only knock-down of BAX and BAK but also the ROS scavenger glutathione diminish cisplatin induced apoptosis. Mitochondrial ROS correlates with mitochondrial content and reduction of mitochondrial biogenesis by knock-down of transcription factors PGC1a or TFAM attenuates both mtROS induction and cisplatin induced apoptosis. Increasing mitochondrial ROS by inhibition or knock-down of the ROS-protective uncoupling protein UCP2 enhances cisplatin induced apoptosis. Similarly, enhancing ROS by high-dose ascorbic acid or $\mathrm{H}_{2} \mathrm{O}_{2}$ augments cisplatin induced apoptosis. In summary, mitochondrial content and the resulting mitochondrial capacity to produce ROS critically determine HGSC cell sensitivity to cisplatin induced apoptosis. In line with this observation, data from the human protein atlas (www.proteinatlas.org) indicates that high expression of mitochondrial marker proteins (TFAM and TIMM23) is a favorable prognostic factor in ovarian cancer patients. Thus, we propose mitochondrial content as a biomarker for the response to platinum-based therapies. Functionally, this might be exploited by increasing mitochondrial content or mitochondrial ROS production to enhance sensitivity to cisplatin based anti-cancer therapies.
\end{abstract}

\section{Introduction}

Platinum compounds, i.e. cisplatin $\left(\left[\mathrm{Pt}\left(\mathrm{NH}_{3}\right)_{2} \mathrm{Cl}_{2}\right]\right)$ or carboplatin $\left(\left[\mathrm{Pt}\left(\mathrm{C}_{6} \mathrm{H}_{6} \mathrm{O}_{4}\right)\left(\mathrm{NH}_{3}\right)_{2}\right]\right)$ are among the most important agents for chemotherapeutic treatment of solid cancers. As such, platinum complexes are currently used as an inherent part of standard therapy for patients with

\footnotetext{
Correspondence: Frank Essmann (frank.essmann@ikp-stuttgart.de)

${ }^{1}$ Dr. Margarete-Fischer-Bosch Institute of Clinical Pharmacology and University of Tuebingen, Stuttgart, Germany

${ }^{2}$ Institute of Cell Biology and Immunology, University of Stuttgart, Stuttgart, Germany

Full list of author information is available at the end of the article.

These authors contributed equally: Walter E. Aulitzky, Frank Essmann

Edited by C. Munoz-Pinedo
}

high-grade serous ovarian cancer (HGSC) ${ }^{1,2}$. Commonly HGSC patients benefit from first-line therapy but eventually experience cancer recurrence. Hence, despite initial response ovarian cancer was, in the United States in 2017, the fifth leading cause of cancer death in women and the 5 -year relative survival rate in patients with HGSC is as low as $46 \%^{3}$. In view of this evident treatment failure a deeper understanding of cellular response to platinum drugs is highly demanded to allow the identification of effective combinations to thwart resistance.

Altered energy metabolism of cancer cells compared to normal tissue was first shown by Otto Warburg ${ }^{4}$. The "Warburg effect" describes "aerobic glycolysis" as a

\section{(c) The Author(s) 2019}

(c) (i) Open Access This article is licensed under a Creative Commons Attribution 4.0 International License, which permits use, sharing, adaptation, distribution and reproduction cc) in any medium or format, as long as you give appropriate credit to the original author(s) and the source, provide a link to the Creative Commons license, and indicate if changes were made. The images or other third party material in this article are included in the article's Creative Commons license, unless indicated otherwise in a credit line to the material. If material is not included in the article's Creative Commons license and your intended use is not permitted by statutory regulation or exceeds the permitted use, you will need to obtain permission directly from the copyright holder. To view a copy of this license, visit http://creativecommons.org/licenses/by/4.0/. 
biochemical phenomenon by which cancer cells generate energy from conversion of glucose to lactate even when properly oxygenated. Later it has become clear that not all tumor cells depend on "aerobic glycolysis" to generate energy as the oxygen supply is a function of the localization of the tumor cell within the tissue ${ }^{5}$. Intracellularly, energy in form of ATP is produced by mitochondria. These pivotal organelles also influence more distant cellular features ranging from stemness to differentiation and cell death (apoptosis) ${ }^{6}$. Apoptosis via the intrinsic signaling pathway crucially depends on mitochondrial release of cytochrome c, which acts as an electron transporter in energy metabolism. The context specific dual function of cytochrome c strikingly illustrates the intimate connection of energy metabolism and apoptosis. Consequently, "metabolic checkpoints" regulate induction of apoptosis and major cell death regulators like BAX and MCL-1 influence mitochondrial metabolism ${ }^{7}$.

Anticancer therapy by chemotherapeutic drugs commonly aims to compromise cellular integrity by damaging nuclear DNA thereby inducing cell death. Less prominent is the fact that mitochondrial DNA (mtDNA), like nuclear DNA (nDNA), is strongly affected by cisplatin. In fact cisplatin adducts of mitochondrial DNA are present at a 300 - to 500 -fold ratio as compared to $\mathrm{nDNA}^{8}$. Consequently, mtDNA-damage is evident in cisplatin treated cells $s^{9}$. The importance of mitochondria is readily evident when analyzing the response to cisplatin in cells with depleted mtDNA ( $\rho^{0}$ cells): Such $\rho^{0}$ cells derived from the cisplatin-sensitive ovarian cancer cell line 2008 acquired resistance to cisplatin induced cell death ${ }^{10}$. In line with cell death critically depending on mtDNA-damage rather than nDNA-damage, apoptosis induction by cisplatin in testicular germ cell tumor cells does not require the nDNA-damage response mediating proteins ATM, ATR, or DNA-PK ${ }^{11}$. Even enucleated colon cancer cells still undergo apoptosis in response to cisplatin ${ }^{12}$.

Since several lines of evidence indicate that tumor sensitivity to cisplatin induced apoptosis is a function of mitochondria, we investigated the role of mitochondria in cisplatin induced apoptosis and elucidate the molecular basis for the involvement of mitochondria. Our analyses clearly show that cells respond to cisplatin by increasing the cellular amount of mitochondria, correlating directly with the sensitivity to cisplatin induced apoptosis. We propose that cisplatin mediated apoptosis driven by mitochondria is caused by cisplatin induced mitochondrial ROS that is detrimental to the cells and that the ROS level is a function of cellular mitochondrial content.

\section{Material and methods}

Cell culture and tumor samples

A2780, IGROV-1, OVCAR-3, OVCAR-4, OVCAR-5, and OVCAR-8 cells were purchased from the ATCC NCI-60 cell panel. All cells were cultivated in RPMI-1640 (Biochrom, Berlin, Germany) supplemented with 10\% FCS and $10 \mathrm{mM}$ L-glutamine. Cell cultures were routinely tested for mycoplasma.

\section{Reagents and experimental conditions}

Cells were preincubated for $2 \mathrm{~h}$ with either $2 \mathrm{mM}$ glutathione (Sigma-Aldrich, Munich, Germany), $50 \mu \mathrm{M}$ zVAD-fmk (Bachem, Bubendorf, Switzerland), $5 \mu \mathrm{M}$ Oligomycin A (BIOZOL, Eching, Germany), $2 \mathrm{mM}$ ascorbic acid (Sigma-Aldrich), and $300 \mu \mathrm{M} \mathrm{H}_{2} \mathrm{O}_{2}$ (Otto Fischar $\mathrm{GmbH}$, Saarbrücken, Germany). Then, cisplatin was added at $10 \mu \mathrm{M}$. Cells were simultaneously incubated with cisplatin and $100 \mu \mathrm{M}$ Genipin (Sigma-Aldrich).

\section{Generation of monoclonal cell lines}

500 cells were seeded into a coated $60 \mathrm{~cm}^{2}$ petri dish and cultured for 10-14 days. Evolving colonies were individually detached by trypsination using a silicon ring, seeded into 24-well plates and expanded.

\section{Detection of apoptosis}

Annexin V-FITC (BD Pharmingen, Heidelberg, Germany) and propidium iodide (Sigma-Aldrich) staining was performed and measured by flow cytometry.

\section{Detection of mitochondrial content}

Cells were stained with $200 \mathrm{nM}$ Mitotracker Green (Thermo Fisher Scientific Inc.) or Acridine Orange 10nonyl bromide (NAO) (Sigma-Aldrich) harvested and analyzed by flow cytometry.

\section{Oxygen consumption rate}

After incubation in the presence or absence of above stated compounds cells were harvested by trypsination and 40.000 cells were seeded into a Seahorse Cell Culture Microplate, centrifuged and analyzed in a Seahorse XF96 Extracellular Flux Analyzer. A Seahorse XF Cell Mito Stress Test was performed with $1 \mu \mathrm{M}$ Oligomycin A, $0.5 \mu \mathrm{M}$ FCCP and $1 \mu \mathrm{M}$ Antimycin A + Rotenone.

\section{Detection of mitochondrial ROS}

Adherent cells were harvested by trypsination, counted and $0.1 \times 10^{6}$ cells were stained with MitoSOX red (Thermo Fisher Scientific Inc.) and analyzed by flow cytometry.

\section{Measurement of cell division rate}

Cells were seeded in a 6-well plate and harvested in 4 consecutive days for cell number determination.

\section{Detection of mitochondrial membrane potential}

Cells were harvested, counted and $0.1 \times 10^{6}$ cells were stained with TMRM (Thermo Fisher Scientific Inc.) and analyzed by flow cytometry. 


\section{siRNA experiments}

Gene expression of BAX, BAK, PGC1 $\alpha$, TFAM, or UCP2 was silenced using siGENOME SMARTpool siRNA (Horizon Discovery Ltd, Cambridge, GB). Control cells were transfected with siGenome Non-Targeting siRNA \#1. In total $72 \mathrm{~h}$ after transfection using DharmaFECT\#1 (Horizon Discovery Ltd), cells were incubated with $10 \mu \mathrm{M}$ cisplatin $48 \mathrm{~h}$. The efficacy of silencing was evaluated by western blot.

\section{Western blot analysis}

Cells were harvested by trypsination, washed in ice cold PBS and lysed in $50 \mathrm{mmol} / \mathrm{l}$ Tris- $\mathrm{HCl}, 250 \mathrm{mmol} / \mathrm{l} \mathrm{NaCl}$, 0.1\% Triton X-100, 5 mmol/l EDTA, pH 7.6, supplemented with cOmplete protease inhibitor cocktail (Roche, Grenzach-Wyhlen, Germany) and PhosSTOP phosphatase inhibitor cocktail (Roche) followed by sonication (50 cycles, $20 \mathrm{sec}$ ). Equal amounts of protein were diluted in $5 x$ Laemmli buffer, heated $\left(95^{\circ} \mathrm{C}, 5 \mathrm{~min}\right.$.) and separated by SDS polyacrylamide gel electrophoresis. Proteins were transferred onto $0.45 \mu \mathrm{m}$ nitrocellulose membrane. Incubation steps were: $1 \mathrm{~h}$ in blocking buffer (5\% non-fat dry milk; $50 \mathrm{mmol} / \mathrm{l}$ Tris-HCl; $125 \mathrm{mM} \mathrm{NaCl}$; $\mathrm{pH} 7.0$ ) with shaking. Washing steps were $4 \times 5 \mathrm{~min}$ in TBST; incubation with primary antibodies was over night at $4{ }^{\circ} \mathrm{C}$, incubation with secondary antibodies was $1 \mathrm{~h}$ with shaking. Primary antibodies were as follows: anti-BAX \#2772, antiBAK \#3814, anti-TFAM \#8076, anti-UCP2 \#89326, and anti-GAPDH (14C10) \#2118 (Cell Signaling, Denvers, MA, USA); anti-PGC1 $\alpha$ \#ab54481 (Abcam, Cambridge, UK).

\section{TCGA data analysis}

Survival data of the TCGA-OV project was downloaded from the TCGA Research Network: http://cancergenome. nih.gov/. Corresponding RNA expression data of the patients was obtained from the Human Protein Atlas Project (v15.0) (https://www.proteinatlas.org/ENSG00000108064TFAM/pathology/tissue/ovarian+cancer) (https://www. proteinatlas.org/ENSG00000265354-TIMM23/pathology/ tissue/ovarian + (cancer) ${ }^{13}$.

\section{Statistical analysis}

Data from at least three independent experiments are expressed as average \pm standard deviation (SD). Statistical analysis was performed using GraphPad Prism 4.0 (GraphPad Software, La Jolla, USA). Changes in paired or unpaired samples were analyzed using two-sided paired or unpaired $t$-test and significance was considered when $p$-value $<0.05\left(" p<0.05\right.$; $\left.{ }^{* *} p<0.01 ;{ }^{* * * *} p<0.001\right)$.

\section{Results}

Sensitivity to cisplatin induced cell death correlates with mitochondrial content

Platinum based chemotherapeutic compounds critically target nuclear DNA integrity. Less well studied is the impact of cisplatin on mitochondria. To clarify the role of mitochondrial functionality in cisplatin-resistance of ovarian cancer cells we first analyzed cellular sensitivity to cisplatin induced cell death in high-grade ovarian cancer cell lines from the NCI-60 panel. Cells from six cell lines (OVCAR-3, OVCAR-4, OVCAR-5, OVCAR-8, IGROV-1, and A2780) were incubated in the presence of $10 \mu \mathrm{M}$ cisplatin for $48 \mathrm{~h}$ and induction of apoptosis was analyzed by Annexin V-FITC/PI staining and flow cytometric analysis (Fig. 1a). Cisplatin efficiently induced cell death in OVCAR-3, OVCAR-4, and IRGOV-1 ( $>60 \%$ cell death) whereas OVCAR-5, OVCAR-8, and A2780 showed $<30 \%$ apoptotic cells in response to cisplatin. Based on these results we grouped the cells in "sensitive" (OVCAR-3, OVCAR-4, and IGROV-1) and "resistant" (OVCAR-5, OVCAR-8, and A2780). To further analyze the role of mitochondria in cisplatin induced cell death we assessed the relative mitochondrial content by staining the cells with Mitotracker Green and subsequent flow cytometric analysis (Fig. 1b). These analyses show that the sensitive cell lines are characterized by higher relative mitochondrial content (mean 1.52) than the resistant cell lines (mean 0.91). To confirm the disparate mitochondrial content of sensitive and resistant cells we detected the mitochondria specific lipid cardiolipin using $10-\mathrm{N}$-nonyl acridine orange (NAO) in flow cytometric analysis (Fig. 1c). Again, a higher relative content of cardiolipin indicating higher mitochondrial content was observed in sensitive cell lines as compared to resistant cell lines. Hence, higher mitochondrial content directly correlates with sensitivity to cisplatin induced apoptosis. As mitochondrial reactive oxygen species are known to be toxic, we next analyzed the cellular levels of mitochondrial reactive oxygen species (mtROS) by MitoSOX red staining (Fig. 1d). Expectedly, the sensitive ovarian carcinoma cell lines were characterized by higher mtROS as compared to the resistant cell lines. We also analyzed in a Seahorse XF96 Extracellular Flux Analyzer the mitochondrial basal oxygen consumption rate (OCR). A higher OCR was detected in the sensitive than in the resistant cell lines (Fig. S1a), which is in concordance with a higher mitochondrial content.

In order to further analyze the impact of mitochondrial content in a genetically constant background we generated clonal sublines from the cisplatin sensitive OVCAR-3 cell line. Despite genetic identity, these subclones show disparate mitochondrial content and disparate sensitivity to cisplatin induced cell death ${ }^{14}$. In agreement with our previous results, these OVCAR-3 subclones display a significant correlation of the relative mitochondrial content and their sensitivity to cisplatin induced apoptosis (Fig. 1e). Similar to the relative mitochondrial content also the OCR correlated with sensitivity to cisplatin induced apoptosis (Fig. 1f). These data show a clear correlation of 


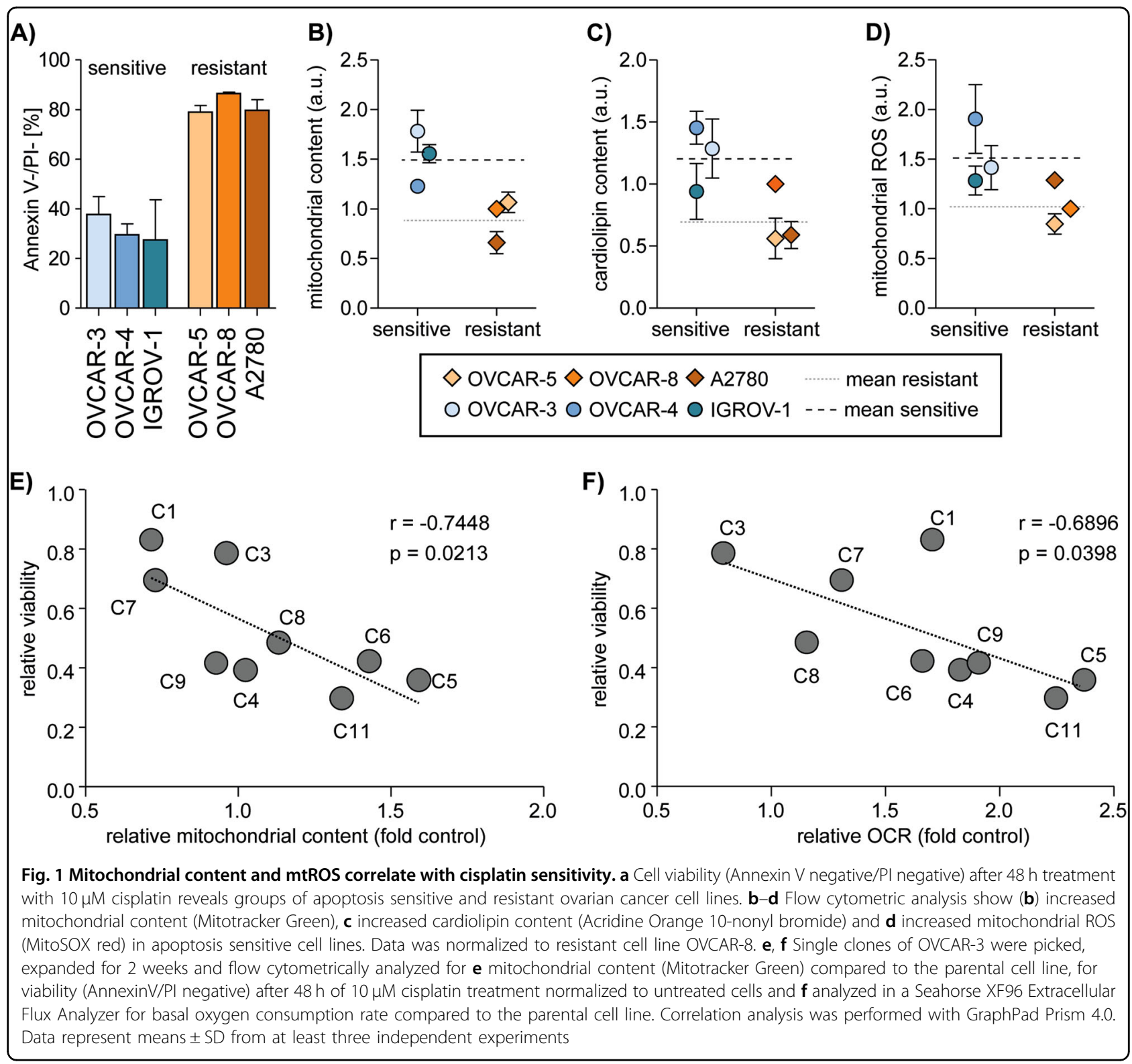

mitochondrial activity and cellular sensitivity to cisplatin induced apoptosis not only in different cell lines but also in genetically similar sublines generated from a common precursor cell line.

\section{Cisplatin induced cell death depends on ROS, BAX\&BAK and caspases but is independent of proliferation of the cells}

Nuclear DNA-damage induction by chemotherapeutic drugs is particularly toxic for rapidly proliferating cells. Intriguingly, we found that the cisplatin sensitive cell lines OVCAR-3, OVCAR-4, and IGROV-1 show a slightly lower proliferation rate than the resistant cell lines OVCAR-5, OVCAR-8, and A2780 (Fig. 2a). Thus, in addition to nuclear DNA-damage induction by cisplatin its known effect to induce production of ROS in target cells might impact on cell death induction ${ }^{15}$. To analyze the relevance of ROS in cisplatin induced cell death we compared viability of cells that had been incubated with cisplatin in the presence or absence of the ROS scavenger glutathione (GSH) (Fig. 2b). Incubation with GSH did not affect the viability of OVCAR- 3 and OVCAR- 4 cells but efficiently enhanced viability of cells incubated with cisplatin: OVCAR-3 (38\% vs $75 \%$ ); OVCAR-4 (35\% vs $67 \%$ ). In an analogous experiment we verified that cisplatin induced cell death is apoptotic rather than necrotic by incubating cells with the pan-caspase inhibitor zVAD-fmk (Fig. 2c). Inhibition of caspase-activity completely blocked cell death induction verifying apoptotic cell death. Acceptedly, apoptosis mediated by the mitochondrial 

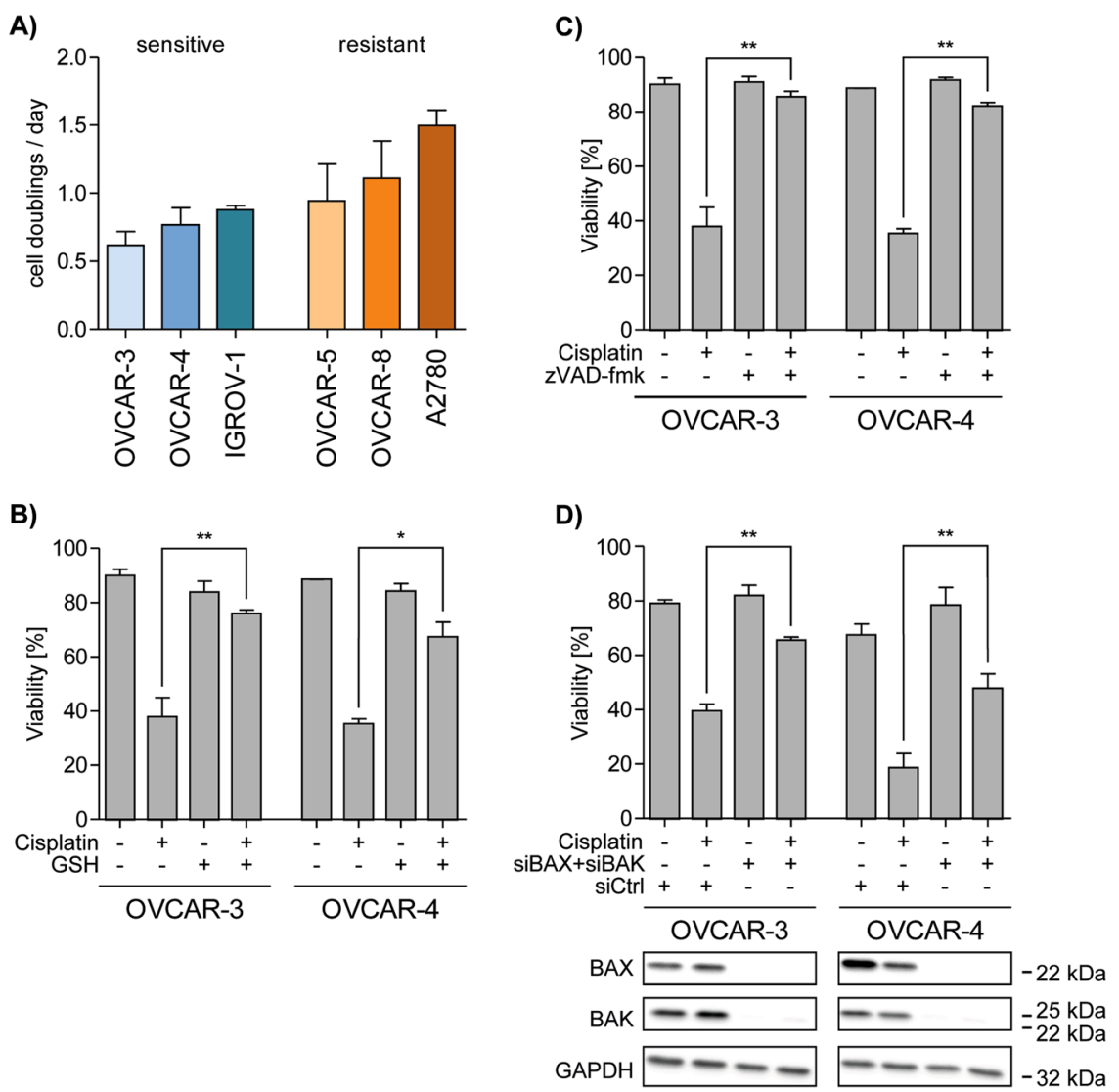

Fig. 2 Cisplatin induces intrinsic and ROS dependent apoptosis. a Division rate was determined by counting cells on 4 consecutive days. $\mathbf{b}$, c Viable (Annexin $V$ negative/PI negative) cells after co-incubation with b $2 \mathrm{mM}$ Glutathion or c $50 \mu \mathrm{M}$ zVAD-fmk and $10 \mu \mathrm{M}$ cisplatin for $48 \mathrm{~h}$. (d, bottom) Western blot analysis of BAX and BAK expression in cells transfected with siRNA targeting BAX and BAK or control siRNA and (d, top) flow cytometric analysis of viability (Annexin $\mathrm{V}$ negative/PI negative) after treatment with $10 \mu \mathrm{M}$ cisplatin for $48 \mathrm{~h}$. Data represent means \pm SD from at least three independent experiments

signaling pathway critically depends on the pore-forming effector proteins BAX and/or BAK ${ }^{16}$. To provide further proof for mitochondria-mediated apoptosis we employed small-interfering RNA to knock-down the effector proteins BAX\&BAK and subsequently analyzed cisplatin induced apoptosis. In line with cell death induction via the mitochondrial apoptosis pathway, knock-down of BAX\&BAK (Fig. 2d, lower panel) efficiently rescued cells from cisplatin induced cell death (Fig. 2d, upper panel). These data show that cell death induction by cisplatin depends on the induction of ROS, the presence of poreforming BAX\&BAK and active caspases.

\section{Cisplatin induces mtROS, enhances mitochondrial content} and increases mitochondrial membrane potential $\Delta \Psi_{\mathrm{m}}$

Cisplatin is a well-known inducer of $\operatorname{ROS}^{17-19}$ but the intracellular origin of ROS production is not fully understood. An important source of ROS in mammalian cells are mitochondria ${ }^{20}$ and especially cisplatin induced ROS was shown to be produced by mitochondria ${ }^{21}$. We analyzed cisplatin induced ROS production in the sensitive cell lines OVCAR-3 and OVCAR-4 and the resistant cell line OVCAR-8 (Fig. 3a). Induction of ROS by cisplatin was detected in all cell lines, however, the sensitive OVCAR-3 and OVCAR-4 cells showed higher ROS production compared to the resistant OVCAR-8 cells under control conditions as well as in response to cisplatin (vertical lines). Similarly, increased ROS was detected by CellROX Green or CellROX Deep Red, which have slightly altered specificity for cellular ROS as compared to MitoSOX (Fig. S1b, c). Because the sensitive cells also showed higher mitochondrial content (Fig. 1b, c) we wondered whether cisplatin induced ROS production also correlated with a further increase in mitochondrial content. Indeed, analysis of mitochondrial content by staining of the cells with Mitotracker Green clearly indicated an increase of mitochondrial content in response to cisplatin in sensitive OVCAR-3 and OVCAR-4 but also in resistant OVCAR-8 cells (Fig. 3b, left panel). Noteworthy, the peak mitochondrial content was consistently higher in sensitive 


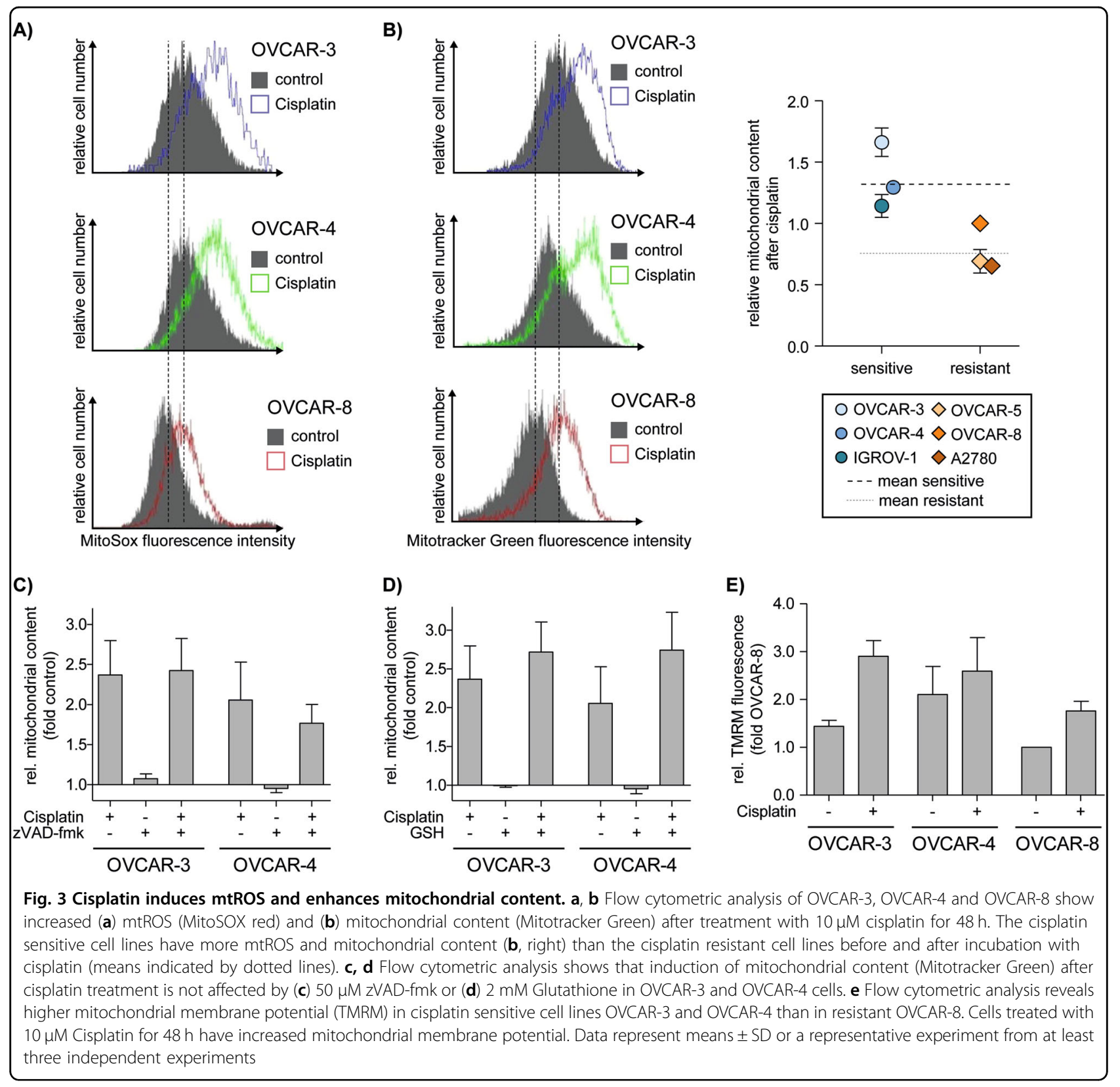

cells as compared to cells resistant to cisplatin induced apoptosis (Fig. 3b, right panel). In order to delineate whether increased mitochondrial content is cause or consequence of cisplatin induced ROS production or apoptosis we again incubated cells with the ROS scavenger GSH or the pan-caspase-inhibitor zVAD-fmk. Subsequent flow cytometric analysis showed that neither GSH nor caspase-inhibition prevents the cisplatin induced increase in mitochondrial content. Hence, we conclude that cisplatin induces an increase of mitochondrial content upstream of ROS production and caspase activity (Fig. 3c, d). In line with upstream induction of mitochondrial content, TMRM staining showed increased overall mitochondrial membrane potential $(\Delta \Psi \mathrm{m})$ in OVCAR-3, OVCAR-4, and OVCAR-8 cells (Fig. 3e). In line with cisplatin induced increase of mitochondrial content, cisplatin also increased the basal OCR and the maximal respiration in OVCAR-3 and OVCAR-8 cells (Fig. S1d). Taken together, cisplatin treatment of ovarian cancer cell lines induces increased mitochondrial content, enhanced OCR and mitochondrial membrane potential and higher levels of mitochondrial ROS. Noteworthy, induction of mitochondrial content is upstream of ROS induction and caspase activation. 


\section{Mitochondrial content and ROS are causally linked to cisplatin induced cell death}

To further elucidate the role of elevated mitochondrial activity in response to cisplatin treatment, we blocked mitochondrial function by the ATP synthase inhibitor Oligomycin A. After $48 \mathrm{~h}$, the OCR was reduced and could not be reduced any further by additional injection of Oligomycin A in the "MitoStress Test" (Supplementary Fig. S2a). Inhibition of ATP synthase during cisplatin treatment prevented the induction of mtROS (Fig. 4a) and also increase of the mitochondrial membrane potential (Supplementary Fig. S2b), whereas mitochondrial content was still amplified (Fig. 4b). Diminished ROS induction was accompanied by significant reduction of cisplatin induced apoptosis (Fig. 4c), showing the importance of increased $\mathrm{mtROS}$ for apoptosis induction.

The mitochondrial transcription factor TFAM drives the mitochondrial transcription machinery ${ }^{22}$ and regulates mitochondrial biogenesis ${ }^{23,24}$. Expression of TFAM was increased in sensitive OVCAR-3 and resistant OVCAR-8 cells after $48 \mathrm{~h}$ incubation with cisplatin which is in line with mitogenesis (Fig. 4d). Mitogenesis associated expression of TFAM is regulated by PGC1 $\alpha$ (Peroxisome proliferator-activated receptor gamma coactivator 1-alpha), the master regulator of mitochondrial biogenesis ${ }^{25}$. A partial knock-down of PGC1 $\alpha$ prevented the cisplatin induced increase of TFAM protein expression (Fig. 4e). Moreover, knock-down of PGC1 $\alpha$ reduced ROS induction after cisplatin treatment (Fig. 4f) and significantly protected sensitive OVCAR- 4 cells from cisplatin induced apoptosis (Fig. 4g). In line, also knockdown of TFAM prevented cisplatin induced apoptosis (Fig. 4h). Overall, blocking mitochondrial function or preventing mitochondrial biogenesis causes a decrease of mtROS induction by which sensitive ovarian cancer cells can be rescued from cisplatin induced cell death.

\section{Mitochondrial content is a prognostic factor for survival of ovarian cancer patients}

The presented data shows that a key element of cisplatin induced cell death is mitochondrial ROS and mitochondrial content. The expression of mitochondrial transcription factor TFAM and Translocase Of Inner Mitochondrial Membrane 23 (TIMM23) reflect mitochondrial content and might therefore be indicative for response of HGSC to cisplatin based therapy, which still is the first-line therapy of ovarian cancer after surgery ${ }^{1,2}$. Hence, it is conceivable that TFAM and TIMM23 expression - as surrogate markers for mitochondrial content - correlate with enhanced overall survival. We investigated TFAM and TIMM23 expression and overall survival in the TCGA-OV (v15.0) and the project data indeed shows significantly $(p<0.005)$ higher 5 -year survival probability for patients with high expression of
TFAM (41\%) or TIMM23 (45\%) as compared to those with low TFAM (28\%) or TIMM23 (28\%) expression (Fig. $5 \mathrm{a}, \mathrm{b})$. Thus, we propose that high expression of mitochondrial proteins like TFAM and TIMM23 are favorable prognostic factors for HGSC patients because their expression correlates with mitochondrial content and mtROS production, which in turn amplifies sensitivity to cisplatin induced apoptosis. Intriguingly, highest expression of TFAM and TIMM23, among all tissues, is found in testis (www.proteinatlas.org; ${ }^{13}$ ) - and testis cancer is commonly cured by cisplatin therapy.

\section{Increase of mtROS overcomes cisplatin resistance}

We show that inhibition of mitochondrial function and mitogenesis enhance the survival of cisplatin sensitive cells and that sensitivity to cisplatin induced apoptosis correlates with mitochondrial content and mtROS. Increased mtROS induces activation of counteracting "uncoupling proteins" (UCP) that decrease mtROS production and mitochondrial membrane potential by mediating leakage of protons through the mitochondrial inner membrane ${ }^{26-28}$. We analyzed proton leakage using the MitoStress Test and found increased proton leakage in response to cisplatin (Supplementary Fig. S3) in OVCAR8 cells. Hence, we thought to exploit these mechanistic insights to improve anti-cancer therapy and asked whether Genipin, an inhibitor of UCP2, enhances cellular sensitivity to cisplatin induced apoptosis. In accordance with the above outlined mechanisms, Genipin significantly increased cisplatin induced mitochondrial ROS in the resistant cell line OVCAR-8 (Fig. 6a) and also enhanced cell death (Fig. 6b). In an alternative approach we aimed to increase cisplatin induced apoptosis by knocking-down the ROS protective UCP2. Also, reduced UCP2 expression clearly resulted in enhanced sensitivity to cisplatin induced apoptosis (Fig. 6c). Increased intracellular ROS acceptedly is also induced by high concentration of ascorbic $\operatorname{acid}^{29,30}$. At $2 \mathrm{mM}$ concentration ascorbic acid caused a minor increase of mtROS levels in OVCAR-8 while ascorbic acid together with cisplatin strongly enhanced mtROS levels (Fig. 6d) resulting in cell death of the resistant OVCAR-8 cells (Fig. 6e). In order to verify that ROS are causally linked to cisplatin induced cell death OVCAR-8 cells were incubated with cisplatin in combination with $\mathrm{H}_{2} \mathrm{O}_{2}$. Although exogenous $\mathrm{H}_{2} \mathrm{O}_{2}$ $(300 \mu \mathrm{M})$ did not significantly affect cell viability, exogenous ROS clearly aided in cisplatin induced cell death (Fig. 6f). Increasing ROS by pharmacological means might potentially sensitize ovarian cancer cells to cisplatin and overcome resistance.

\section{Discussion}

Platinum compounds have been used in anti-cancer therapy for decades and their damaging activity on nDNA 


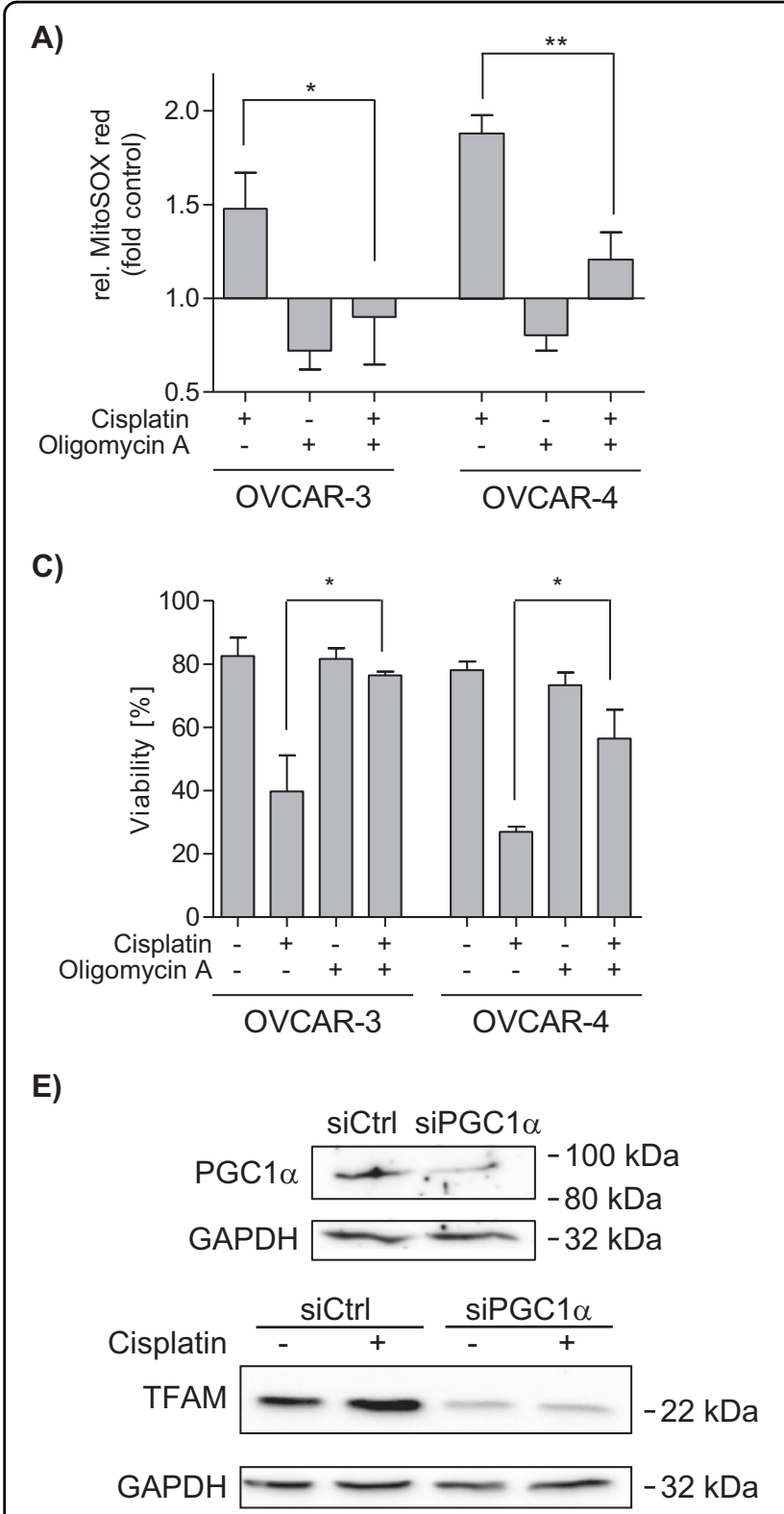

B)

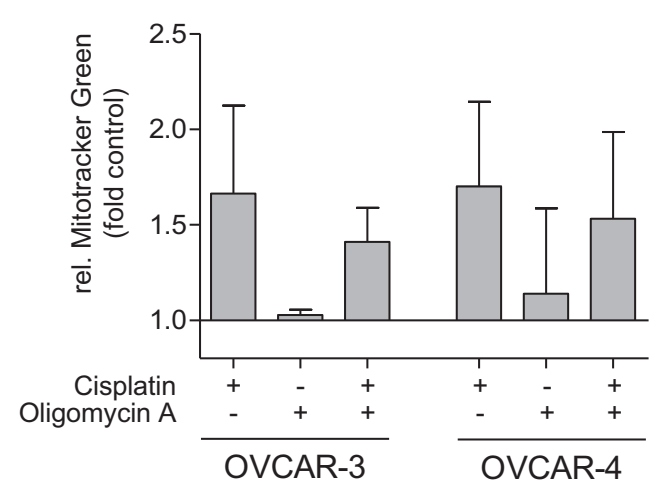

D)

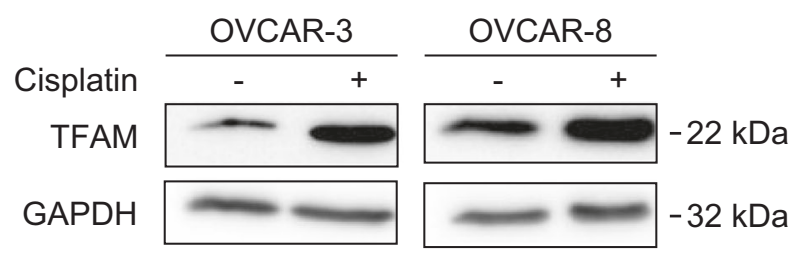

F)

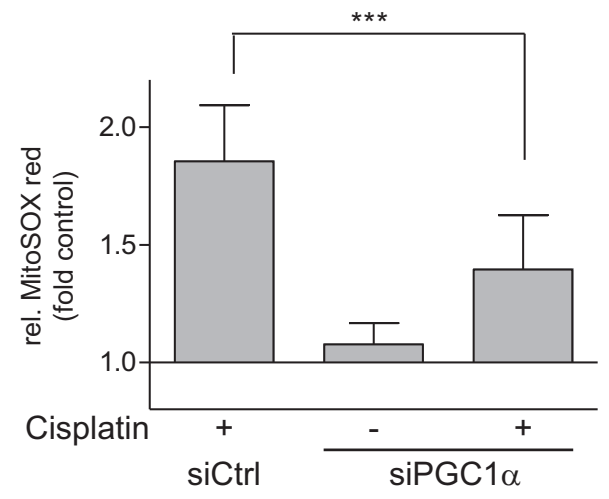

G)
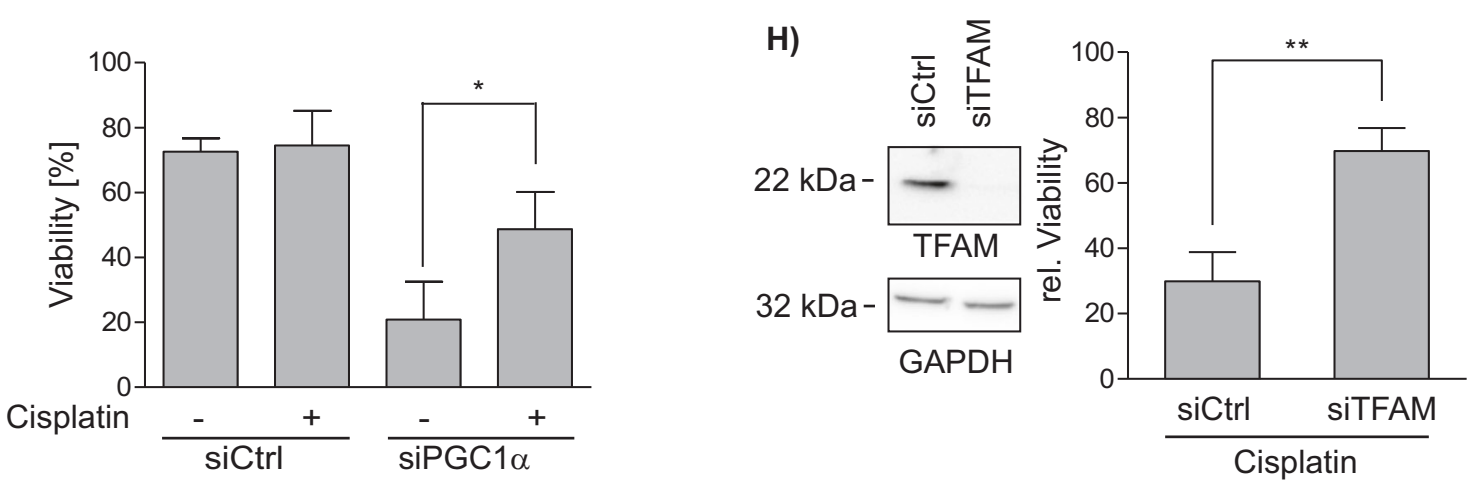

Fig. 4 (See legend on next page.) 
(see figure on previous page)

Fig. 4 Reduction of mtROS enhances survival after cisplatin treatment. a-c Flow cytometric analysis of OVCAR-3 and OVCAR-4 after cOtreatment with ATP synthase inhibitor Oligomycin A $(5 \mu \mathrm{M})$ and $10 \mu \mathrm{M}$ cisplatin for $48 \mathrm{~h}$. a Oligomycin A reduces cisplatin mediated mtROS induction (MitoSOX red) while b mitochondrial content (Mitotracker Green) is unaffected. c Oligomycin A increases viability (Annexin V negative/PI negative) after cisplatin incubation. $\mathbf{d}$ Western blot analysis of OVCAR-3 and OVCAR-8 cells after treatment with $10 \mu \mathrm{M}$ cisplatin for $48 \mathrm{~h}$ shows increased TFAM protein expression. $\mathbf{e}-\mathbf{g}$ siRNA mediated knockdown of PGC1a in OVCAR-4. e Expression of PGC1a (top) and TFAM (bottom) are decreased in transfected OVCAR-4 cells. f Flow cytometric analysis shows decreased mtROS induction (MitoSox red) in PGC1a silenced cells after treatment with $10 \mu \mathrm{M}$ cisplatin for $48 \mathrm{~h}$. g Flow cytometric analysis of cell viability (Annexin $\mathrm{V}$ negative/PI negative) reveals PGCA1 a knockdown mediated enhanced survival after treatment with $10 \mu \mathrm{M}$ cisplatin for $48 \mathrm{~h}$. (h, left) siRNA mediated knockdown of TFAM in OVCAR-4 cells (h, right) is associated with enhanced viability (Annexin $\mathrm{V}$ negative/PI negative) in a flow cytometric analysis after treatment with $10 \mu \mathrm{M}$ cisplatin for $48 \mathrm{~h}$. Data represent means $\pm \mathrm{SD}$ or a representative experiment from at least three independent experiments

A)
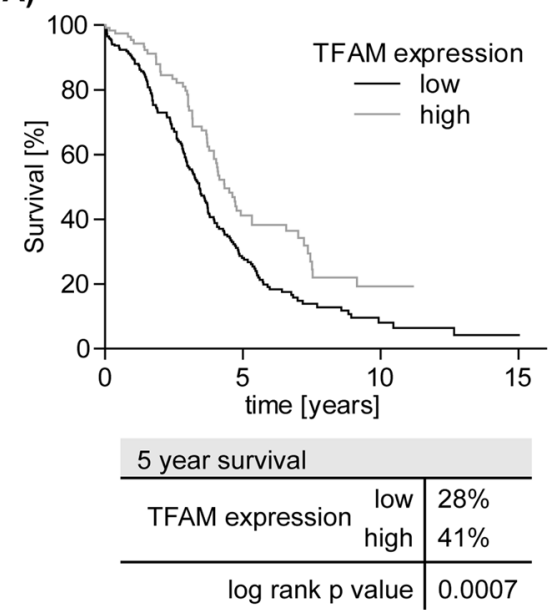

B)
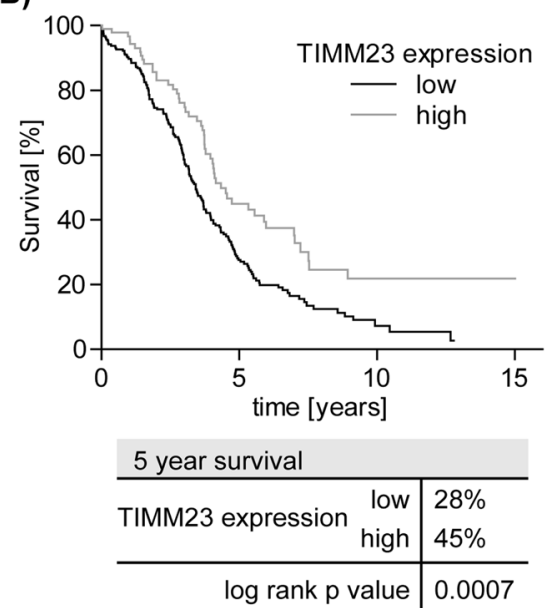

Fig. 5 Mitochondrial content correlates with better $\mathbf{5}$-year survival of OVCAR patients. a, b Survival data analysis of the TCGA-OV project. a Kaplan-Meier plot of patients with high or low TFAM expression with cut off at 10.82 FPKM. b Kaplan-Meier plot of patients with high or low TIMM23 expression with cut off at 61.81 FPKM. Data obtained from the TCGA Research Network (http://cancergenome.nih.gov) and the human protein atlas (www.proteinatlas.org)

is well characterized. However, focusing on the nuclear DNA-damage response does not show the entire effect of cisplatin on cells given that there is an additional pool of DNA in cellular organelles, i.e. mitochondria. Undoubtedly, mitochondria are a central component of intrinsic apoptosis signaling. Not surprisingly mitochondria play an important role in chemo-resistance ${ }^{31-33}$. Interestingly, cisplatin induced damage of $\mathrm{mtDNA}^{8,10}$ affects mitochondrial function and enhances cell death ${ }^{21}$. Therefore, we aimed to investigate the effect of cisplatin on mitochondria and the role of mitochondria in the cellular response to cisplatin in ovarian cancer cells. For the first time we present evidence that cisplatin sensitive ovarian cancer cell lines (OVCAR-3, OVCAR-4, and IGROV-1) show higher mitochondrial content and more mtROS than less sensitive or resistant cell lines (OVCAR-5, OVCAR-8, and A2780). Furthermore, clonal and hence most likely isogenic sublines of cisplatin sensitive OVCAR-3 cells confirmed these results, because even in these sublines the relative mitochondrial content and OCR correlate with susceptibility to cisplatin induced cell death. These results are in line with observations that cisplatin resistant ovarian cancer cell line $\mathrm{C} 13$ present with reduced mitochondrial function and enhanced dependency on glycolysis and the pentose phosphate pathway than their sensitive counterpart $2008^{10,33}$. Consequently, depletion of mitochondrial DNA in $\rho^{0}$ cells mediates resistance to cisplatin induced cell death ${ }^{8,10}$.

Cisplatin induces intrinsic apoptosis that depends on pore-forming pro-apoptotic $\mathrm{Bcl}-2$ family proteins BAX\&BAK and caspase activation. More importantly, we find that cisplatin mediated activation of the intrinsic apoptosis pathway depends on mitochondrial ROS. In line, cisplatin induced apoptosis even independent of nuclear DNA is illustrated by the fact that testicular germ cell tumor cells still undergo cisplatin induced apoptosis in the absence of key mediators of DNA damage response, such as ATM, ATR, or DNA-PK ${ }^{11}$. Furthermore, 


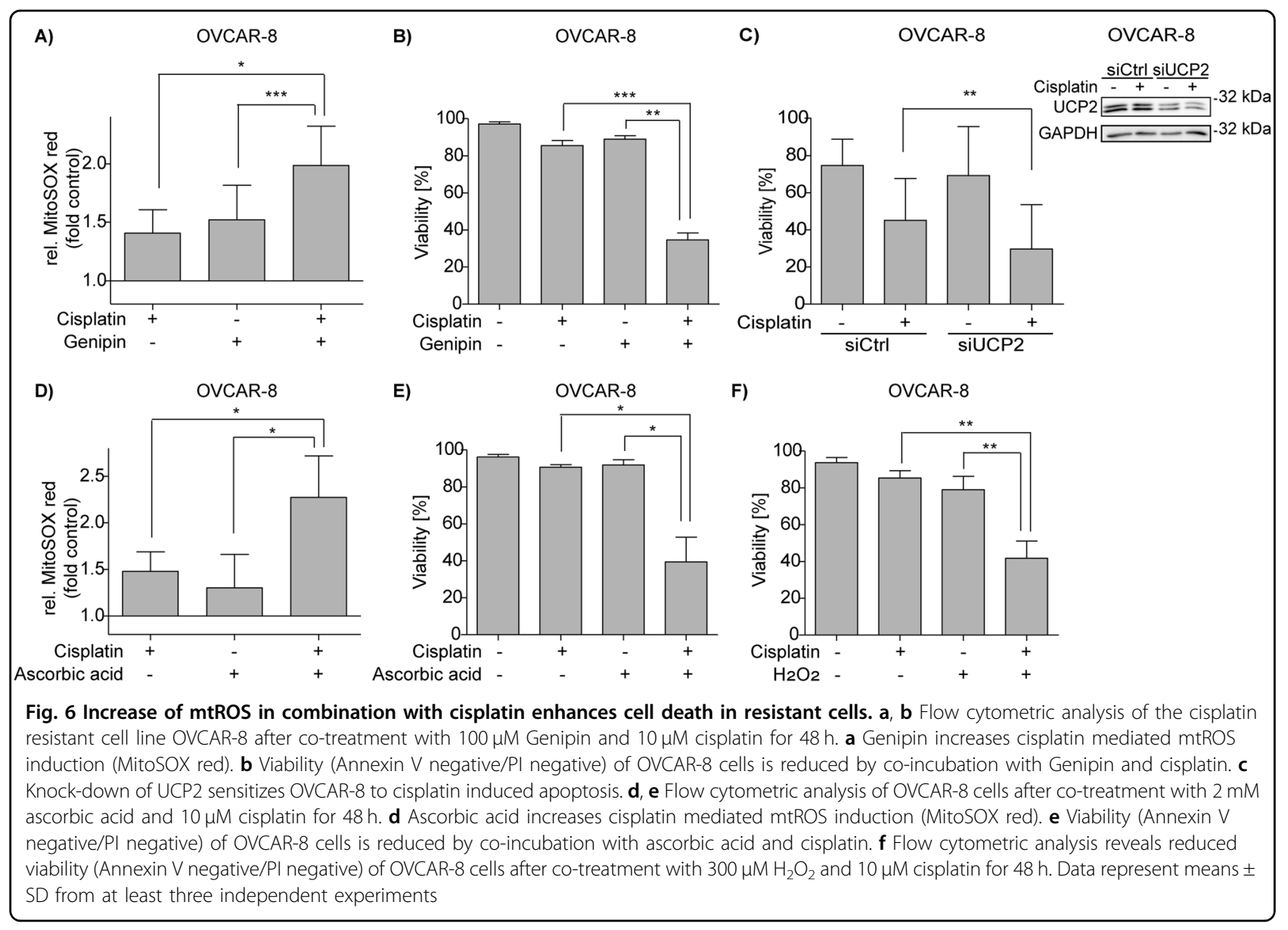

enucleated colon cancer cells undergo apoptosis in response to cisplatin ${ }^{12}$, thus placing mitochondria into the focus of cisplatin induced apoptosis.

Mechanistically, mitochondria likely play a dual role in cisplatin induced apoptosis: while BAX\&BAK mediated permeabilization and release of cytochrome $\mathrm{c}$ is indispensable for the induction of the effector phase of apoptosis, we show a critical function of cisplatin induced increase of mitochondrial content and $\mathrm{mtROS}^{21}$ upstream of BAK\&BAK oligomerization. We present evidence by various methods that cisplatin enhances cellular mitochondrial content (e.g. Fig. 3b, S1d). However, a remaining question still is whether (a) a subpopulation of damaged mitochondria produces increased mtROS or (b) the higher amount of mtROS originates from basal mtROS production by increased mitochondrial content. We here show that increased mitochondrial content correlates with increased mtROS and enhanced sensitivity to cisplatin induced cell death. We propose that mitochondrial content and cisplatin induced mitogenesis influence sensitivity to cisplatin induced apoptosis. We confirm this by showing that (a) blocking ATP synthase with Oligomycin A blocks mtROS production and (b) blocking mitochondrial biogenesis by knock-down of PGC1 $\alpha$ or TFAM reduces cisplatin induced cell death. Reduced mitochondrial content alleviates mtROS and renders sensitive cells resistant to cisplatin induced cell death. On the other hand, increase of mitochondrial ROS by Genipin mediated blocking of UCP2 or knock-down of UCP2, treatment with high doses of ascorbic acid or the application of $\mathrm{H}_{2} \mathrm{O}_{2}$ enhance cisplatin induced cell death. The role of mtROS in cisplatin induced apoptosis is illustrated by reduced toxicity of carboplatin that does induce far less $m t_{R O S}{ }^{21}$. Production of $m t R O S$ can be enhanced by tuning mitochondrial metabolism, e.g. by dichlor acetate that shift mitochondria from glycolysis to glucose oxidation ${ }^{34}$ resulting in enhanced sensitivity to cisplatin $^{21}$. Likewise, cultivation of cells in glucose-free medium complemented with galactose increases mitochondrial activity ${ }^{35}$. Therefore, cultivation in galactosesupplemented medium, not surprisingly, renders resistant ovarian cancer cells sensitive to cisplatin induced cell death $^{36}$.

Cisplatin treatment impairs the integrity $\mathrm{mtDNA}^{8,37}$, induces mtROS and thereby causes reduced energy production by generation of dysfunctional mitochondria ${ }^{15}$. 


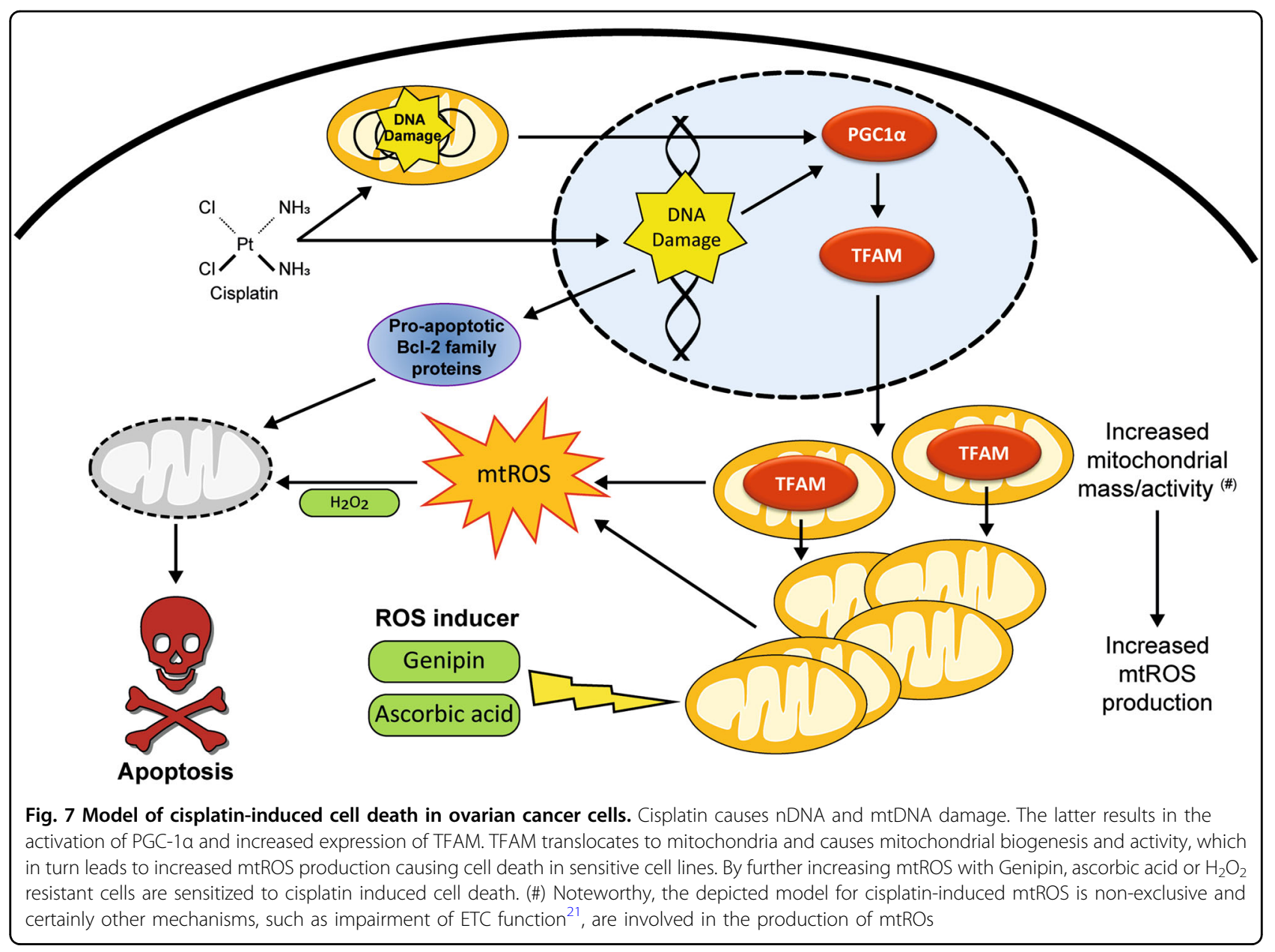

Mitochondrial dysfunction and mtROS in turn enhance mitochondrial biogenesis by inducing the expression of PGC1 $\alpha$, nuclear respiratory factors 1 and 2 (Nrf1, Nrf2), and $\mathrm{TFAM}^{38}$. Since intact mitochondria during respiration partially convert consumed oxygen to $\mathrm{mtROS}^{39}$ it is obvious that the cisplatin induced increased mitochondrial content inevitably results in higher levels of mtROS. However, previous studies show that cisplatin induced mtROS results from inhibition of transcription of mtDNA-encoded proteins, resulting in impairment of ETC function ${ }^{21}$ (Fig. 7 (\#)). In any case, because the level of mtROS positively influences sensitivity to cisplatin induced apoptosis induction it is worthwhile to investigate whether pharmacological compounds that increase mitochondrial biogenesis and/or mtROS or decrease the activity of the anti-oxidative machinery are suitable to enhance the efficacy of cisplatin based anti-cancer therapy or even overcome resistance in ovarian cancer patients (Fig. 7). This aim might be achieved by modern pharmacological intervention using genipin (Fig. 6a, b) or more unconventional approaches such as high-dose ascorbic acid (Fig. 6d, e).
Undoubtedly, the relevance of mitochondrial content for therapy response is evidenced by RNA expression analyses available in TCGA-OV data that show superior overall survival of ovarian cancer patients with high expression of the mitochondrial proteins TFAM and TIMM23. Also, in line with the proposed role of mitochondrial content and mtROS production in response to cisplatin based anticancer therapy, analysis of the TCGAOV data indicates a favorable therapy response of patients with low expression of $\mathrm{UCP}^{40}$, a protein that reduces production of mtROS. In summary, we provide solid evidence for a highly relevant function of mitochondria in cisplatin based anti-cancer therapy beyond and yet intricately entangled with their indispensable role in the intrinsic apoptosis signaling pathway. The mechanistic insights presented here establish mitochondria as new marker structures for the prediction of therapy response. Furthermore, improved therapy response might be achievable by combining cisplatin based drugs with substances that impair efficient function of ROS-protective mechanisms, either by pharmacological or physiological means such as hyper-oxygenation. 


\section{Acknowledgements}

We are grateful to Kerstin Willecke for excellent technical assistance. This study was supported by the Robert Bosch Stiftung (project O2).

\section{Author details}

${ }^{1}$ Dr. Margarete-Fischer-Bosch Institute of Clinical Pharmacology and University of Tuebingen, Stuttgart, Germany. ${ }^{2}$ Institute of Cell Biology and Immunology, University of Stuttgart, Stuttgart, Germany. ${ }^{3}$ Department of Hematology and Oncology, Robert-Bosch-Hospital, Stuttgart, Germany

\section{Conflict of interest}

The authors declare that they have no conflict of interest.

\section{Publisher's note}

Springer Nature remains neutral with regard to jurisdictional claims in published maps and institutional affiliations.

Supplementary Information accompanies this paper at (https://doi.org/ 10.1038/s41419-019-2081-4).

Received: 27 May 2019 Revised: 22 October 2019 Accepted: 23 October 2019

Published online: 07 November 2019

\section{References}

1. Morgan, R. J. et al. Ovarian Cancer, Version 1.2016, NCCN Clinical Practice Guidelines in Oncology. J. Natl Compr. Canc. Netw. 14, 1134-1163 (2016).

2. Ledermann, J. A. et al. Newly diagnosed and relapsed epithelial ovarian carcinoma. Ann. Oncol. 24 (Suppl 6), vi24-vi32 (2013).

3. Siegel, R. L., Miller, K. D. \& Jemal, A. Cancer Statistics, 2017. CA Cancer J. Clin. 67, 7-30 (2017).

4. Warburg, O. On the origin of cancer cells. Science 123, 309-314 (1956).

5. Danhier, P. et al. Cancer metabolism in space and time. Biochim. Biophys. Acta 1858, 556-572 (2017).

6. Vega-Naredo, I. et al. Mitochondrial metabolism directs stemness and differentiation in P19 embryonal carcinoma stem cells. Cell Death Differ. 21, 1560-1574 (2014).

7. Green, D. R., Galluzzi, L. \& Kroemer, G. Cell biology. Metabolic control of cell death. Science 345, 1250256 (2014).

8. Yang, Z. et al. Cisplatin preferentially binds mitochondrial DNA and voltage-dependent anion channel protein in the mitochondrial membrane of head and neck squamous cell carcinoma. Clin. Cancer Res. 12 5817-5825 (2006).

9. Podratz, J. L. et al. Cisplatin induced mitochondrial DNA damage in dorsal root ganglion neurons. Neurobiol. Dis. 41, 661-668 (2011).

10. Montopoli, M. et al. "Metabolic Reprogramming" in ovarian cancer cells resistant to cisplatin. Curr. Cancer Drug Targets 11, 226-235 (2011).

11. Gutekunst, M. et al. p53 hypersensitivity is the predominant mechanism of the unique responsiveness of testicular germ cell tumor (TGCT) cells to cisplatin. PLOS ONE 6, e19198 (2011).

12. Berndtsson, M. et al. Acute apoptosis by cisplatin requires induction of reactive oxygen species but is not associated with damage to nuclear DNA. Int. J. Cancer 120, 175-180 (2007).

13. Uhlen, M. et al. A pathology atlas of the human cancer transcriptome. Science 18, 357 (2017).

14. Márquez-Jurado, S. et al. Mitochondrial levels determine variability in cell death by modulating apoptotic gene expression. Nat. Commun. 9, 389 (2018).
15. Choi, Y.-M. et al. Mechanism of cisplatin-induced cytotoxicity is correlated to impaired metabolism due to mitochondrial ROS generation. PLOS ONE 10 e0135083 (2015).

16. Dewson, G. \& Kluck, R. M. Mechanisms by which Bak and Bax permeabilise mitochondria during apoptosis. J. Cell Sci. 122, 2801-2808 (2009).

17. Jin, J. et al. Protective effect of Wuzhi tablet (Schisandra sphenanthera extract) against cisplatin-induced nephrotoxicity via Nrf2-mediated defense response. Phytomedicine 22, 528-535 (2015).

18. Marzano, C. et al. Inhibition of thioredoxin reductase by auranofin induces apoptosis in cisplatin-resistant human ovarian cancer cells. Free Radic. Biol. Med. 42, 872-881 (2007).

19. Rybak, L. Dose dependent protection by lipoic acid against cisplatin-induced ototoxicity in rats. Toxicol. Sci. 47, 195-202 (1999).

20. Murphy, M. P. How mitochondria produce reactive oxygen species. Biochem. J. 417, 1-13 (2009).

21. Marullo, R. et al. Cisplatin induces a mitochondrial-ROS response that contributes to cytotoxicity depending on mitochondrial redox status and bioenergetic functions. PLOS ONE 8, e81162 (2013).

22. Shi, $Y$. et al. Mammalian transcription factor $A$ is a core component of the mitochondrial transcription machinery. Proc. Natl Acad. Sci. USA 109 16510-16515 (2012).

23. Picca, A. \& Lezza, A. M. S. Regulation of mitochondrial biogenesis through TFAM-mitochondrial DNA interactions. Mitochondrion 25, 67-75 (2015).

24. Jornayvaz, F. R. \& Shulman, G. I. Regulation of mitochondrial biogenesis. Essays Biochem. 47, 69-84 (2010)

25. Fernandez-Marcos, P. J. \& Auwerx, J. Regulation of PGC-1a, a nodal regulator of mitochondrial biogenesis. Am. J. Clin. Nutr. 93, 8845-890S (2011).

26. Echtay, K. S., Murphy, M. P., Smith, R. A. J., Talbot, D. A. \& Brand, M. D. Superoxide activates mitochondrial uncoupling protein 2 from the matrix side. Studies using targeted antioxidants. J. Biol. Chem. 277, 47129-47135 (2002).

27. Echtay, K. S. et al. Superoxide activates mitochondrial uncoupling proteins Nature 415, 96-99 (2002).

28. Krauss, S., Zhang, Y. \& Lowell, B. B. The mitochondrial uncoupling-protein homologues. Nat. Rev. Mol. Cell Biol. 6, 248-261 (2005).

29. Yun, J. et al. Vitamin C selectively kills KRAS and BRAF mutant colorectal cancer cells by targeting GAPDH. Science 350, 1391-1396 (2015).

30. Takemura, Y. et al. High dose of ascorbic acid induces cell death in mesothelioma cells. Biochem. Biophys. Res. Commun. 394, 249-253 (2010).

31. Guerra, F., Arbini, A. A. \& Moro, L. Mitochondria and cancer chemoresistance. Biochim. Biophys. Acta Bioenerg. 1858, 686-699 (2017).

32. Wolf, D. A. Is reliance on mitochondrial respiration a "chink in the armor" of therapy-resistant cancer? Cancer Cell 26, 788-795 (2014).

33. Catanzaro, D. et al. Inhibition of glucose-6-phosphate dehydrogenase sensitizes cisplatin-resistant cells to death. Oncotarget 6, 30102-30114 (2015).

34. Woo, D. K. et al. Mitochondrial genome instability and ROS enhance intestinal tumorigenesis in APC(Min/+) mice. Am. J. Pathol. 180, 24-31 (2012).

35. Dott, W., Mistry, P., Wright, J., Cain, K. \& Herbert, K. E. Modulation of mitochondrial bioenergetics in a skeletal muscle cell line model of mitochondrial toxicity. Redox Biol. 2, 224-233 (2014).

36. Wintzell, M. et al. Repeated cisplatin treatment can lead to a multiresistant tumor cell population with stem cell features and sensitivity to 3bromopyruvate. Cancer Biol. Ther. 13, 1454-1462 (2012).

37. Garrido, N. Cisplatin-mediated impairment of mitochondrial DNA metabolism inversely correlates with glutathione levels. Biochem. J. 414, 93-102 (2008). al.

38. Lee, H-C. \& Wei, Y.H. Mitochondrial biogenesis and mitochondrial DNA maintenance of mammalian cells under oxidative stress. Int. J. Biochem. Cell Biol. 37, 822-834 (2005).

39. Chance, B., Sies, H. \& Boveris, A. Hydroperoxide metabolism in mammalian organs. Physiol. Rev. 59, 527-605 (1979).

40. Kawanishi, M. et al. Expression of UCP2 is associated with sensitivity to platinum-based chemotherapy for ovarian serous carcinoma. Oncol. Lett. 15 9923-9928 (2018). 